\title{
Weighty matters
}

\section{A successful year for the Large Hadron Collider has culminated in its first lead-ion collisions.}

CERN's Large Hadron Collider has passed another milestone. This striking image, showing the outcome of a collision between two high-energy lead ions, was one of the first recorded when the collider switched over from proton collisions to 'heavy ion' running last month.

Such a transition was always part of the LHC timetable. ALICE - one of the four huge detectors sited in the ring of the collider, and the apparatus that captured this image - is dedicated to the measurement of heavy-ion collisions; two other, generalpurpose detectors, ATLAS and CMS, are also capable of probing this physics. It's a phenomenal environment: the capture, processing and analysis of proton collisions is challenging; but in the collisions of lead ions (whose mass is more than 200 times greater than that of protons) as many as a few thousand particles may emerge at once from the meltdown of the two lead ions.

The intention is to study the formation of quark-gluon plasma, the temperature achieved at the collision point being sufficient for the protons and neutrons of the lead nuclei to break down into their constituent quarks and gluons. Studies performed at lower energies, using gold nuclei, at the Relativistic Heavy Ion Collider at Brookhaven National Laboratory, New York, have indicated that quark-gluon plasma may not behave quite as had been expected. Now the LHC detectors will probe further.

As the physics studies begin in earnest, the LHC itself deserves a moment of acknowledgement. After the false start of 2008, when the failure of a single splice caused extensive damage to a sector of the machine, operation with proton beams began again in 2009. In March 2010, the attempt to achieve collisions with proton energies of a record $3.5 \mathrm{TeV}$ succeeded in a matter of hours. Now the transition from colliding beams of protons to physics with beams of lead ions has been managed in only four days. That record belies the complexity of this machine, which has been twenty years in the making. It is, however, a signal of the talent and skill of the physicists and engineers who designed, built and now operate it.

Heavy-ion running of the LHC will continue this month; collisions of protons and the search for supersymmetry and that elusive Higgs boson - will then resume

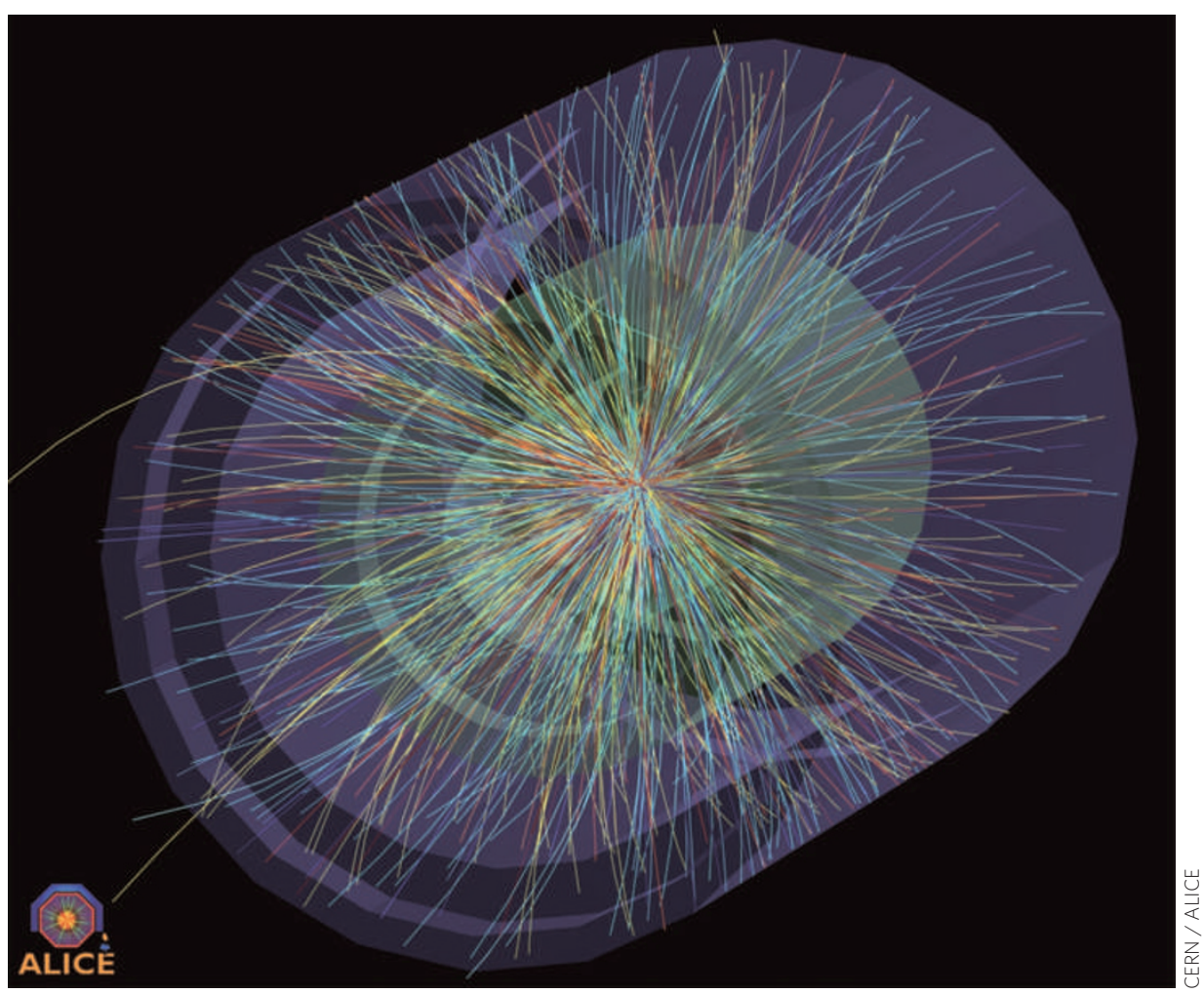

Big bang: a collision of lead ions captured by the ALICE detector at CERN.

in February 2011, after a short winter shut-down, possibly at an increased beam energy of $4 \mathrm{TeV}$. An extensive period of shut-down, to prepare the LHC to meet its design criterion of $7 \mathrm{TeV}$ per beam, is scheduled throughout 2012 and into early 2013. That hiatus in the running of the LHC has motivated physicists at Fermilab's Tevatron, near Chicago, to push for an extension to their own programme.

Commissioned in 1983, the Tevatron collides $\mathrm{TeV}$ beams of protons and antiprotons. Although the LHC already has the energy advantage, the Tevatron has the benefit of data - lots of it. As well as discovering the top quark, the CDF and $\mathrm{D} \varnothing$ detectors at the Tevatron have conducted some of the most extensive explorations of particle interactions, and there are some tantalizing anomalies in their data at present. The Tevatron will in time be entirely superseded by the LHC, but as the LHC will really only hit the heights after its 2012/2013 shut-down period, the planned closure of the Tevatron in September 2011 has unsurprisingly started to seem a little inopportune.

Three more years, through to 2014, would enable the Tevatron detectors to effectively double their current datasets (http://arxiv. org/abs/1011.1532). That should iron out any anomalies and create a meaningful legacy to LHC physicists of data and analyses that are in many ways complementary to those that will be generated at CERN. However, there are only so many particle physicists in the world, and there are concerns over resourcing a continuation of the Tevatron programme when manpower is most vital to capture and understand data from the LHC. And although there has been some high-level endorsement in the USA, whether the necessary funding will be forthcoming is as yet unclear the next hurdle will be the US President's Department of Energy budget request for the financial year 2012, due in February 2011.

Meanwhile, there should be corks popping in the LHC control centre when the last 'fill' of 2010 comes to an end. It has truly been a miraculous year. 